\title{
LASER ACCELERATORS: EXPERIMENTS, COMPUTATIONS AND PROSPECTS
}

\author{
C. JOSHI \\ Electrical Engineering Department \\ University of California, Los Angeles, CA 90095-1594
}

\begin{abstract}
Ever since the research on laser-based particle acceleration concepts began in earnest about a dozen years ago, there has been very significant progress in the field of Advanced Accelerator Research. Proof-of-principle experiments that demonstrate the physical principle of the various schemes have now been carried out. These experiments are guided by state-of-the-art computer simulations made possible by the recent revolution in computing power and techniques. The research is poised to move on to the next level where it will address both physics and technology issues that are at the very forefront of beam science.
\end{abstract}

\section{INTRODUCTION}

I will start off by giving a general motivation for advanced accelerator research. In most of the advanced accelerator schemes the accelerating structures are far smaller than the present structures driven by r.f. in the $\mathrm{s}$ or $\mathrm{x}$ band range. The wavelength of the structure is typically in the $1 \mathrm{~mm}$ to $10 \mu \mathrm{m}$ range which means that these structures are powered by either optical frequency sources or short bunches of electrons. The beams produced by such structures are similarly expected to be ultrashort: in the subpicosecond to a few femtosecond range. The production, manipulation (preservation) and diagnostics of such ultrashort bunches represent the forefront of research in beam physics. Once such bunches become available, their interaction with matter or radiation will open new opportunities in the physical sciences. On the technology front the holy grail of advanced accelerator $\mathrm{R} \& \mathrm{D}$ is to produce a practical, table-top, $1 \mathrm{GeV}$ electron accelerator that can deliver a peak current in the 10-100 Amp range. Finally, one hopes that in the long run, advanced accelerator R \& D will yield a new technology for a future high energy collider.

The key physics milestones for any advanced accelerator scheme are as follows:

1. Demonstration of the physical principle for acceleration.

2. Demonstration of energy gain for externally injected electrons.

3. Demonstration of high gradients ( say $>1 \mathrm{GeV} / \mathrm{m}$ ).

4. Acceleration at this high gradient over a significant length (say 1 meter) or alternately an energy gain of 1 $\mathrm{GeV}$.
5. Demonstration of a reasonably small energy spread and emittance.

6. Demonstration of staging.

It is gratifying to note that all the three laser acceleration schemes that I shall be discussing have demonstrated 1) and 2) above and laser-plasma schemes have in addition demonstrated 3). The second generation laser accelerator research then is moving on to topics 4), 5) and 6). It should be noted that technology and economy issues are at present not being addressed with the same degree of seriousness as the physics milestones.

I shall concentrate now on 3 laser-based particle acceleration schemes. The first is the laser driven plasma wave accelerators. ${ }^{(1)}$ The second is known as the inverse free-electron laser accelerator (IFEL) ${ }^{(2)}$ while the third is the inverse Cherenkov accelerator (ICA). ${ }^{(3)}$ In all accelerators the accelerating particles obey the equation of motion $^{(4)}$

$$
\frac{\mathrm{d} y}{\mathrm{~d} z}=\frac{\mathrm{e}(\mathbf{v} \bullet \mathbf{E})}{\mathrm{mc}^{3}}
$$

which means that there are basically two ways a particle moving in the $\mathrm{z}$ direction can gain energy. First, if the electric field has a component $\mathrm{E}_{\mathrm{z}}$ in the direction of motion of the particle and second if the particle is given a small but finite velocity component in the transverse direction so that it can interact with a transverse component $\mathrm{E}_{\perp}$ of the electric field. Most acceleration schemes including the various laser-plasma schemes and the inverse Cherenkov scheme rely on the first method (i.e., $v_{z} E_{z}$ ) whereas the IFEL relies on the second (i.e., $\left.\mathrm{v}_{\perp} \mathrm{E}_{\perp}\right)$.

\section{PHYSICAL PRINCIPLES}

In the laser-plasma acceleration schemes the transverse electric field of a laser is transformed into a longitudinal electric field of a space charge wave using a plasma. ${ }^{(1)}$ Physically this is accomplished as follows. In the so-called Laser Wake Field Accelerator (LWFA) $)^{(5)}$ a short laser pulse roughly $\pi \mathrm{c} / \omega_{\mathrm{p}}$ long excites a wake in a plasma. This happens because the gradient of the intensity associated with the shape of the pulse exerts a force called the ponderomotive force on the plasma electrons which are pushed away from the regions of high intensity to the regions of lower intensity. After the pulse passes the electrons snap back because of the space charge force of the ions and set up an oscillation. 
In the Plasma Beat Wave Accelerator (PBWA), ${ }^{(6)}$ two co-propagating laser pulses $\left(\omega_{0}, \mathrm{k}_{\mathrm{o}}\right)$ and $\left(\omega_{1}, \mathrm{k}_{1}\right)$ of much lower intensity than required for LWFA are injected into a plasma which satisfy the resonance condition $\omega_{0}-$ $\omega_{1}=\omega_{\mathrm{p}}$. This once again excites a plasma wave via the ponderomotive force.

In the self-modulated laser wake field accelerator ${ }^{(7)}$ (SMLWFA), an intense but much longer single frequency laser pulse is used to excite the plasma wave. The excitation mechanism is the Raman Forward Scattering Instability (RFS) $)^{(8)}$ which generates a whole band of Stokes and anti-Stokes e.m. waves by scattering from noise fluctuations in the plasma at $\omega_{\mathrm{p}}$. These in turn beat with the original wave to provide the ponderomotive buckets that reinforce the plasma wave. In all three schemes, the plasma oscillation in the 1D limit is purely electrostatic with the longitudinal electric field given by $\varepsilon\left(\mathrm{n}_{\mathrm{e}}\right)^{1 / 2} \mathrm{~V} / \mathrm{cm}$ where $\varepsilon$ is the level of density modulation and $n_{e}$ is the plasma electron density in $\mathrm{cm}^{-3}$. Thus for a plasma density in the range $10^{16}<\mathrm{n}_{\mathrm{e}}<10^{18} \mathrm{~cm}^{-3}$ and $\varepsilon=$ 0.1 one can expect $10^{9}<\mathrm{E}(\mathrm{v} / \mathrm{m})<10^{10}$.

In the inverse Cherenkov accelerator the laser beam is first slowed down in a gaseous dielectric and then made to interact with the particle at the Cherenkov angle $\theta_{c}$ such at $\beta \cos \theta_{c}=1 / n$ where $n$ is the refractive index of the gas. In the zero order approximation if $\beta$ and $\cos \theta_{c}$ do not change, the field seen by the particle remains constant in time. The particle sees an electric field $\mathrm{E}$ sin $\theta_{c} \cos \varphi$ which either accelerates or decelerates the particle depending on the sign of the phase angle $\varphi$. Unfortunately, there is a limit to the maximum accelerating field that can be expected in the ICA scheme. This is given by the breakdown threshold of the gas used to slow the laser down. If extremely short pulses are used, then the gas breakdown will occur because of tunnel ionization mechanism which becomes significant when the laser field produces a potential drop roughly corresponding to the ionization potential across the Bohr radius. This limits the laser intensity to less than $10^{14}$ $\mathrm{W} / \mathrm{cm}^{2}$ for a $\mathrm{CO}_{2}$ laser in hydrogen which in turn means that the maximum accelerating field will be limited to less than $500 \mathrm{MeV} / \mathrm{m}$.

Finally, in the IFEL the particle propagates along the axis of a static magnetic wiggler. The $\underline{\mathrm{v}} \times \underline{\mathrm{B}}$ force gives rise to a perpendicular component of the velocity of the particle which now can interact with the transverse field a co-propagating laser. A different way of looking at how this energy exchange can happen is as follows: The combined action of the laser $\left(\omega_{0}, \mathrm{k}_{\mathrm{o}}\right)$ and a static wiggler field $\left(\omega_{0}, k_{w}\right)$ on the electrons results in a ponderomotive wave. The ponderomotive wave has a phase velocity slightly less than $\mathrm{c}$ since $\mathrm{v}_{\mathrm{ph}}=\omega_{\mathrm{o}} /\left(\mathrm{k}_{\mathrm{o}}+\mathrm{k}_{\mathrm{w}}\right)=\mathrm{c} /(1+$ $\left.\mathrm{k}_{\mathrm{w}} / \mathrm{k}_{\mathrm{o}}\right)$. This ponderomotive well can trap and continuously accelerate electrons. However, for this to happen, the resonance condition which relates the electron energy to the wiggler and laser parameters must be maintained. This is given by $\gamma_{\mathrm{R}}=\left(1+\mathrm{a}_{\mathrm{w}}\right)^{1 / 2}\left(\lambda_{\mathrm{w}} / 2 \lambda_{\mathrm{o}}\right)^{1 / 2}$ where $\mathrm{a}_{\mathrm{w}}$ is the normalized vector potential.

\section{CURRENT STATUS OF PLASMA ACCELERATORS}

\section{Laser Wake Field Accelerator}

Experiments are being carried out at several laboratories around the world on LWFA. ${ }^{(9)}$ The most ambitious current effort is the JAERI/KEK/U. Tokyo collaboration in Japan. ${ }^{(10)}$ This collaboration has put together an impressive laboratory facility which combines a high repetition rate $2 \mathrm{TW}$, Ti-Saphire, $\mathrm{T}^{3}$ laser and a 17 $\mathrm{MeV}$ linac. Their experimental arrangement is shown in Fig. 1.

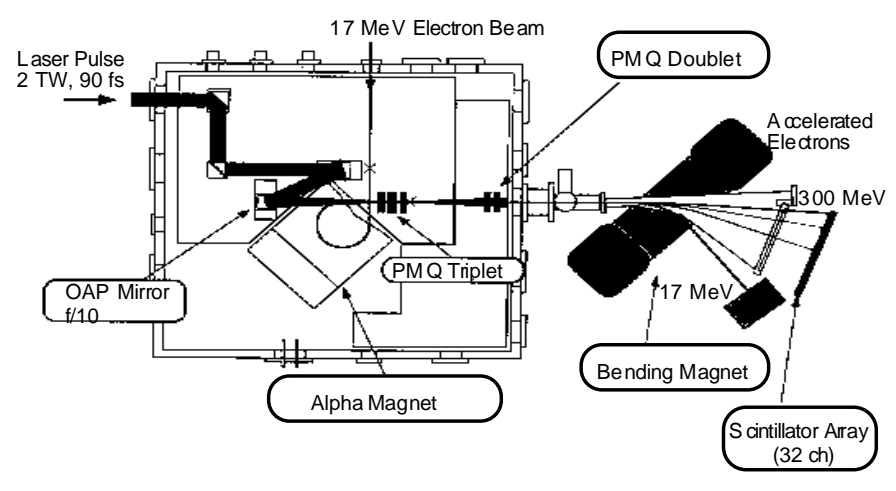

Figure 1. Experimental Set-up for Laser Wakefield Acceleration: KEK-JAERI-U. Tokyo

They have used the technique of frequency domain interferometry to measure the plasma density oscillation in a helium plasma that was produced by the laser. From this they clearly observe a relativistic plasma wave of the correct periodicity and infer a density fluctuation of $\varepsilon=$ 0.15 or a longitudinal field of $4 \mathrm{GeV} / \mathrm{m}$. They have also measured the electron spectra exiting the plasma and have found that when $17 \mathrm{MeV}$ electrons are injected externally into the plasma, electrons up to $100 \mathrm{MeV}$ at 2 Torr and $200+\mathrm{MeV}$ at 20 Torr are observed. To explain the observed maximum energies at the inferred gradients (from spectral interferometry) a coherent plasma wave must exist over many centimeters. Side on images of the plasma taken by the same group do show a plasma that is emitting light over this distance but its not clear if the laser beam is truly self guided or whether there is an alternative explanation for these images. The maximum laser power of $1.8 \mathrm{TW}$ in these experiments is below the critical power for relativistic self-focusing.

\section{Plasma Beat Wave Accelerator}

The most definative experiments have been done by the UCLA group. ${ }^{(11)}$ Using a 2 frequency, $\mathrm{CO}_{2}$ laser they 
have succeeded in accelerating a $2 \mathrm{MeV}$ beam of electrons up to $30 \mathrm{MeV}$ in about $1 \mathrm{~cm}$ giving an average gradient of $2.8 \mathrm{GeV} / \mathrm{m}$. Similar results are reported by a group at AECL in Canada. ${ }^{(12)}$ Furthermore, using a $1 \mu \mathrm{m}$ laser operating on two lines, a group at Ecole Polytechnique ${ }^{(13)}$ has also demonstrated acceleration of externally injected electrons using this technique. A group at Osaka University ${ }^{(14)}$ in Japan also used a two frequency $\mathrm{CO}_{2}$ laser. They observed the acceleration of plasma electrons without any external injection. It is clear that the beat wave acceleration is ready to move on to the next phase of research. The goal of the second generation experiments on the PBWA will be the demonstration of acceleration of a high quality, high current electron beam at the high accelerating gradients that have already been demonstrated. ${ }^{(15)}$ The UCLA group has an ambitious program that proposes to accelerate electrons to $100 \mathrm{MeV}$ while maintaining a small energy spread and emittance. The parameters for their experiment are shown in Table 1. They propose to use a synchronized photoinjector as a source of high current and low emittance injection beam. In order to bunch this beam on a sub 100 micron scale they are considering several bunching schemes.

$\begin{array}{lc}\text { Maximum Energy } & 100 \mathrm{MeV} \\ \text { Total number of particles accelerated } & 10^{8} \\ \text { Number of bunches per macrobunch } & 1-4 \\ \text { Energy spread } & <5 \% \\ \text { Emittance (normalized) } & 10 \pi . \mathrm{mm} . \mathrm{mrad} \\ \text { Accelerated peak current } & 10-100 \mathrm{~A} \\ \text { Acceleration gradient } & 3 \mathrm{GeV} / \mathrm{m} \\ \end{array}$

Table 1. The parameters for UCLA $100 \mathrm{MeV}$ PBWA experiment.

Figure 2 shows the results of computer simulations when a $15 \mathrm{MeV}$ electron beam is injected into the plasma beat wave without and with prebunching. Without prebunching electrons are injected at all phases of the plasma wave whereas in the prebunched case a $60 \mathrm{fs}$ bunch is injected into a $1 \mathrm{ps}$ (300 $\mu \mathrm{m}$ wavelength) plasma wave. The plasma wave has a Lorentzian axial profile with a peak amplitude of $30 \%$ and a diameter of $500 \mu \mathrm{m}$. The initial emittance of the injected beam is $3 \pi \mathrm{mm}$ mrad. One can see that when a prebunched beam is injected more particles are accelerated to high energy as expected and the energy spread is much smaller. This is more clearly shown in Fig. 3. There is a clear peak at approximately $110 \mathrm{MeV}$ with the phase space of particles above $75 \mathrm{MeV}$ showing an emittance of $10 \pi \mathrm{mm} \mathrm{mrad}$.

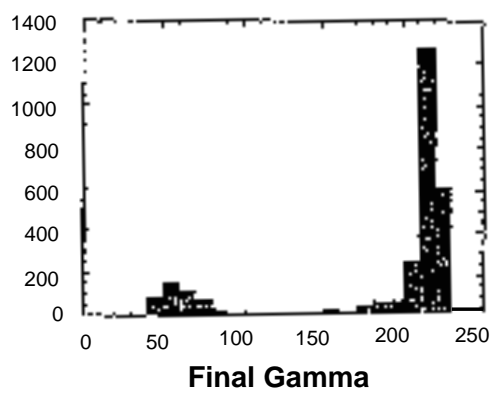

(a)

\section{E $>75 \mathrm{MeV} / \mathrm{e}=10 \pi \cdot \mathrm{mm} . \mathrm{mrad}$}

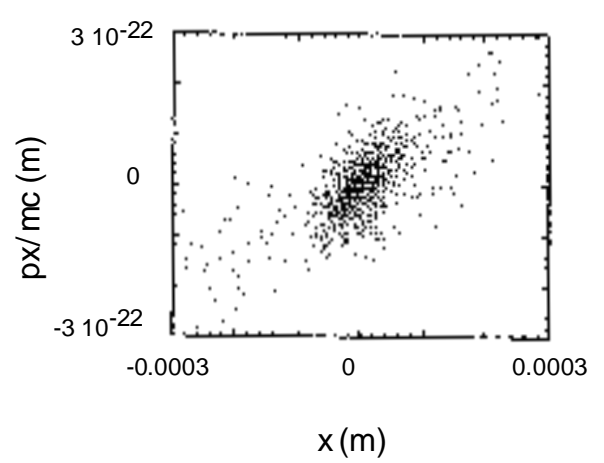

(b)

Figure 2.

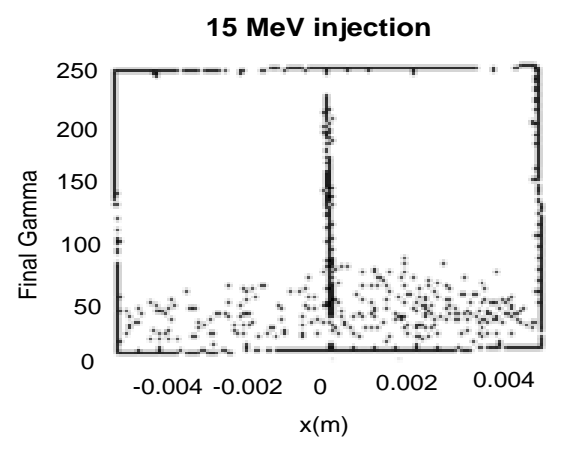

(a)

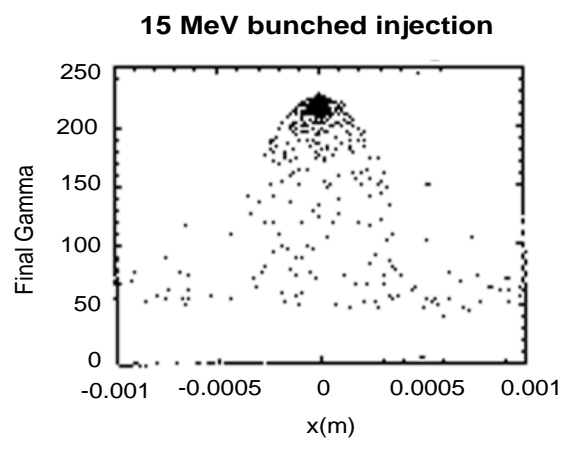

(b)

Figure 3. 


\section{Self-Modulated Laser Wake Field Accelerator}

This scheme has received a lot of attention in the last three years. This is because chirped pulse amplification lasers have become commonplace. Many laboratories around the world have a terawatt class, subpicosecond laser suitable for the studies of SMLWFA. For instance experiments are underway at the Rutherford Laboratory (RAL) ${ }^{(16)}$, NRL ${ }^{(17)}$, Michigan $^{(18)}$ and JAERI ${ }^{(19)}$ on this scheme. The most dramatic results come from the Imperial College/UCLA/Ecole Polytechnique collaboration at the RAL in England. Using a $20 \mathrm{TW}, 1$ ps, $1 \mu \mathrm{m}$ laser this group observed acceleration of selftrapped electrons up to $100 \mathrm{MeV}$ in a $1.5 \times 10^{19} \mathrm{~cm}^{-3}$ density helium plasma. ${ }^{(20)}$ The plasma wave length was simultaneously measured to be about $600 \mu \mathrm{m}$. An energy gain of $100 \mathrm{MeV}$ in $600 \mu \mathrm{m}$ implies an average acceleration gradient of $166 \mathrm{GeV} / \mathrm{m}, 3$ orders of magnitude greater than what is obtained in current r.f. linacs. An interesting aspect of this experiment is that the maximum electron energies observed were larger than expected from the 2D, dephasing limit for externally injected electrons in a plasma wave. Furthermore, the distance over which the electrons were accelerated was greater than the dephasing distance. The explanation to this apparent contradiction is that what was being observed was the energy gain of selftrapped electrons and not externally injected electrons. In the wavebreaking process, the plasma wave amplitude is large enough to trap the main body of the distribution function of the electrons. This causes severe beam loading of the buckets. As the electrons are accelerated, the effective phase velocity of the later bucket speeds up since these buckets are formed as a consequence of the superposition of the original wave field and the wake-field induced by the accelerating electrons. Consequently some trapped particles in the later bucket continue to be accelerated by an accelerating field even though they have gone beyond the normal dephasing length.

What is the future for the SMLWFA scheme? In the near term, availability of 50-100 TW, $0.5 \mu \mathrm{m}$ lasers would imply that we will quite likely see this scheme yielding the first $1 \mathrm{GeV}$ energy gain of any advanced acceleration scheme. This is indeed a very exciting prospect. The eventual practicality of this scheme depends on whether one can phase-lock and inject extremely short bunches to the plasma wave. The plasma waves are sub 10 micron in wavelength so micron size bunches will be required as in the IFEL and ICA schemes.

Recently an all optical injector ${ }^{(21)}$ that is particularly suitable for plasma accelerators has been proposed. In this scheme one intense laser excites the plasma wave while a second even shorter pulse laser $\left(\tau \ll \tau_{\text {plasma }}\right)$ causes local "breaking" of the wave providing the electrons at the correct phase to be accelerated.

The SMLWFA experiments have also shown trantilizing evidence for self-guiding of these very powerful laser pulses. ${ }^{(22)}$ Self-guiding increases the interaction length of the laser with the plasma. However, it remains to be seen if it is a practical way to extend the interaction length of a plasma accelerator since both the plasma density and the laser intensity can vary greatly inside the self-guided channel.

\section{Inverse Free Electron Laser (IFEL)}

There has been a successful IFEL acceleration experiment $^{(23)}$ at the Brookhaven National Laboratory's Accelerator Test Facility. ${ }^{(24)}$ The $\mathrm{CO}_{2}$ laser, the wiggler and the electron beam parameters are given in Table 2.

\begin{tabular}{|c|c|c|c|}
\hline \multicolumn{4}{|l|}{$\mathrm{e}^{-}$beam } \\
\hline & Injection Energy & 40.0 & $\mathrm{MeV}$ \\
\hline & Exit Energy & 42.3 & $\mathrm{MeV}$ \\
\hline & $\mathrm{N}$ (bunch) & $10^{9}$ & $\mathrm{e}^{-}$ \\
\hline & $\Delta \mathrm{E} / \mathrm{E}($ one $\sigma)$ & $\pm 3.10^{-3}$ & \\
\hline & Emittance (one $\sigma$ ) & $7.10^{-8}$ & m.rad \\
\hline \multicolumn{4}{|l|}{ Wiggler } \\
\hline & Wiggler Length & 0.47 & $\mathrm{~m}$ \\
\hline & Period Length, $\lambda_{\mathrm{w}}$ & $2.89-3.14$ & $\mathrm{~cm}$ \\
\hline & Wiggler Gap & 4.0 & $\mathrm{~mm}$ \\
\hline & Field max. (at $6 \mathrm{kA}$ ) & 10 & $\mathrm{kG}$ \\
\hline & Exc. Pulse, 1/2 sin. & 200 & $\mu \mathrm{sec}$ \\
\hline \multicolumn{4}{|c|}{$\mathrm{CO}_{2}$ Laser } \\
\hline & Power & $10^{9}$ & Watts \\
\hline & Pulse (FWHM) & 300 & psec \\
\hline & Max. Field, $\mathrm{E}_{\mathrm{o}}$ & $0.7810^{-3}$ & $\mathrm{MV} / \mathrm{m}$ \\
\hline & Guide Loss, $\alpha$ & 0.05 & $\mathrm{~m}^{-1}$ \\
\hline & $\mathrm{R}_{\mathrm{o}}$, Guide Radius & 1.4 & $\mathrm{~mm}$ \\
\hline
\end{tabular}

Table 2. IFEL Accelerator Experiment, Phase I

(Accelerator Test Facility, Brookhaven National Lab.)

The $\mathrm{CO}_{2}$ laser beam is guided in a saphire waveguide of $2.8 \mathrm{~mm}$ diameter to keep it interacting with the electron beam over the entire wiggler length. The wiggler design is very novel. An easily stakable, variable period, fast excitation driven wiggler is made by using geometrically alternating substacks of Venadium Permandur ferromagnetic laminations, interspaced with conductive nonmagnetic laminations. Although the wiggler period varied, the magnetic field strength is kept constant. They clearly observed the IFEL interaction. In the best case electron energy gain $\Delta \mathrm{P} / \mathrm{P}$ of $2 \%$ was observed for a laser power of less than $0.5 \mathrm{GW}$. This group intends to carry out a parametric study of the IFEL interaction with electron energy, laser intensity, magnetic field strength and laser spot size as variable parameters. In the future this group would like to attempt staging two IFEL modules. ${ }^{(25)}$

An interesting byproduct of the IFEL acceleration experiment is the bunching the accelerating electrons experience on the optical wavelength scale. Efforts are underway at BNL to measure the microbunching in the IFEL experiment. ${ }^{(26)}$ 
A proof-of-principle ICA experiment ${ }^{(27)}$ has been carried out at BNL's ATF using once again their $\mathrm{CO}_{2}$ laser and the synchronized electron beam. A doubleinterferometer optical system is used to convert the $\mathrm{CO}_{2}$ beam into a radially polarized beam which is sent in a gas cell containing 2 atmospheres of hydrogen gas using an axicon. The axicon converges the light so that it interacts with the e-beam at the correct phase matching angle. The experimental parameters are given in Table 3.

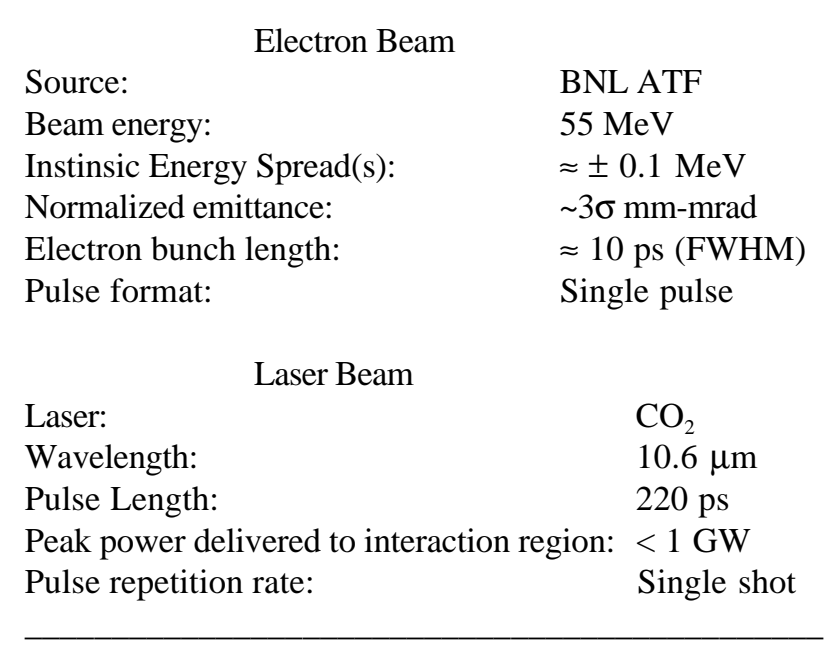

Table 3. Typical ICA Experiment System Parameters

The electron beam energy is measured downstream of the gas cell using a dipole spectrometer. The electrons fall on a phosphor screen and are viewed using an image intensifier and CCD camera. Since the electrons are uniformly distributed over the laser phase both acceleration and energy loss are observed. They have observed an approximately $3.7 \mathrm{MeV}$ energy gain over a $12 \mathrm{~cm}$ interaction length for $0.5 \mathrm{GW}$ laser power, which gives a gradient of $31 \mathrm{MeV} / \mathrm{m}$.

The next phase of the ICA experiment ${ }^{(28)}$ involves using the IFEL as a prebuncher to inject microbunches into the ICA for more efficient acceleration. The length of the gas cell will be increased to $27 \mathrm{~cm}$ for an energy gain of $100 \mathrm{MeV}$. At the same time the IFEL and ICA experiments are being integrated, the laser is being upgraded to one terawatt level. Using $250 \mathrm{GW}$ of $\mathrm{CO}_{2}$ will lead to an accelerating gradient of $370 \mathrm{MeV} / \mathrm{m}$ in the next phase of ICA experiments.
As can be seen from the above discussions, there has been a tremendous progress in the laser-acceleration field. The future of this field looks very exciting with plasma schemes on the threshold of achieving $1 \mathrm{GeV}$ energy gain, IFEL demonstrating microbunching and a combined IFEL/ICA experiment likely to demonstrate staging and phase-locking of charged particles at optical frequencies.

\section{ACKNOWLEDGMENTS}

I wish to thank Drs. Nakajima, A. Dangor, Kimura and van Steenbergen for their input to this paper. This work was supported by DOE grant number DE-AS0392ER40727.

\section{REFERENCES}

1. J. M. Dawson, Scientific American 45, 267 (1979).

2. C. Pellegrini in Laser Acceleration of Particles, (AIP Conf. Proc. No. 91, ed., P. Channell, 1982).

3. J. R. Fontana, R. Pantell, J. Appl Phys. 54, 4285 (1962).

4. J. D. Jackson, Classical Electrodynamics, Ed. J. Wiley (1962).

5. T. Tajima and J. M. Dawson Phys. Rev. Lett. 43, 267 (1979).

6. C. Joshi et al., Nature 311, 525 (1984).

7. P. Sprangle et al., Phys. Rev. Lett. 69, 2200 (1992).

8. C. Joshi et al., Phys. Rev. Lett. 47, 1285 (1981).

9. J. R. Marqués et al., Phys. Rev. Lett. 76, 3566 (1996).

C. Sider et al., Phys. Rev. Lett 76, 3570 (1996).

10. K. Nakajima, private communication.

11. C. Clayton et al., Phys. Rev. Lett. 70, 37 (1993). M. Everett et al., Nature 74, 1355 (1995).

12. N. Ebrahim, J. Appl. Phys. 76, 7645 (1994).

13. F. Amiranoff et al., Phys. Rev. Lett. 74, 5220 (1995).

14. Y. Kitagawa et al., Phys. Rev. Lett. 68, 48 (1992).

15. C. Joshi, Proceedings of the 3rd IAPS meeting, Vol. 5, Japan (1996).

16. A. Modena et al., Nature 377, 608 (1995).

17. A. Ting et al., Phys. Rev. Lett (1996).

18. D. Umstadter et al., Science 76, 2073 (1996).

19. Nakajima et al., 74, 4426 (1995).

20. D. Gordon et al., submitted to Phys. Rev. Lett.

21. D. Umstadter et al., Phys. Rev. Lett. 76, 2073 (1996).

22. C. Clayton et al., Phys. Rev. Lett. to be submitted.

23. A. van Steenbergen et al., Phys. Rev. Lett. 77, 2690 (1996).

24. I. Ben-Zvi, private communication.

25. A. van Steenbergen, private communication.

26. I. Ben-Zvi, private communication.

27. W. Kimura et al., (AIP Conf. Proc., Nov. 325, 1994) p. 131.

28. W. Kimura and A. van Steenbergen, private communication. 